\title{
Case study with natural substances on the various options to identify and categorise endocrine disruptors
}

Ulrike Schuhmacher-Wolz ${ }^{1 *}$, Jens-Uwe Voss ${ }^{2 \dagger}$ and Klaus Schneider ${ }^{1+}$

*Correspondence: ulrike.schuhmacher@fobig.de

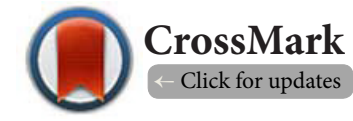

'These authors contributed equally to this work.

${ }^{1}$ Research and Advisory Institute for Hazardous Substances (FoBiG), Freiburg, Germany.

${ }^{2}$ Toxicological Advisory Services, Müllheim, Germany.

\begin{abstract}
Background: Substances with endocrine disrupting properties are under special consideration in European Union regulations. In 2014 the European Commission published a roadmap which presented four possible options for identifying endocrine disruptors (ED). A further option was proposed by the chemical and crop protection industry.

Methods: This case study investigated how natural substances (genistein, caffeine, vitamin D3, sucrose) would possibly be classified under these different options when the focus is on EATS (estrogenic, androgenic, thyroid and steroidogenic) mediated effects on basis of published data.

Results: For sucrose no evidence for endocrine disrupting activity could be identified. The phytoestrogen genistein as well as caffeine would possibly be classified as ED, but caffeine only if potency is not regarded. Vitamin D3 does not elicit EATS mediated adverse effects in vivo.

Conclusion: This screening approach reveals that substances for which safe use in humans is known from decades of experience might possibly be classified as endocrine disruptors with yet unknown regulatory consequences. The examples show that additional criteria beyond the WHO/IPCS definition for ED might be critical for the final classification of a substance as ED.
\end{abstract}

Keywords: Endocrine disruptors, natural substances, classification criteria, screening, caffeine, sucrose, genistein, vitamin D3

\section{Introduction}

In June 2016 the European Commission (EC) published its long awaited draft criteria for the identification of endocrine disruptors (ED). This was the result of a long lasting process initiated by the demand of the biocidal product regulation (Regulation (EU) No 528/2012) and the plant protection products regulation (Regulation (EC) No 1107/2009) to set out scientific criteria for the determination of endocrine disrupting properties by December 2013.

The publication of the criteria was preceded by an extensive scientific debate. In 2014 the EC published a roadmap which presented four possible options for identifying ED (see Table 1). This was followed by a public consultation, and an impact assessment on health, socio-economic and environmental impacts of the four options published in June 2016 [1]. During the impact assessment the Joint Research Centre (JRC) of the European Commission performed a screening exercise with about 700 example substances mainly regulated under the Plant Protection Product Regulation, Biocidal Product Regulation and the REACH Regulation. Natural substances were outside the scope of the JRC project.

However, in future scenario thinking, it could be possible that also natural substances will be involved in the ED discussion, substances like sugars, isoflavones, vitamins, or alkaloids like caffeine are naturally present and also intentionally added to e.g., food or cosmetics. Therefore, the European Chemical Industry Council (Cefic) and the European Crop Protection Association (ECPA) decided to focus on such natural substances and set up a case study. It was not the intention of this project to question the proven safe use of these substances, but to assess the consequences of the four different draft EC options for categorizing ED if applied to natural substances. Additionally, an evaluation option recommended by industry and addressing additional aspects for the evaluation (e.g., lead toxic effects, 
Schuhmacher-Wolz et al., Journal of Toxicology and Health 2017,

http://www.hoajonline.com/journals/pdf/2056-3779-4-2.pdf

doi: $10.7243 / 2056-3779-4-2$

Table 1. EC options presented in the roadmap from $06 / 2014$ to identify endocrine disruptors.

\begin{tabular}{ll}
\hline EC Option & $\begin{array}{l}\text { Criteria for identification of endocrine } \\
\text { disruptors }\end{array}$ \\
\hline EC Option 1 & $\begin{array}{l}\text { Application of interim criteria provided in } \\
\text { legislation: } \\
\text { Identification of ED on basis of their } \\
\text { classification (carcinogenic category 2 and } \\
\text { toxic for reproduction category } 2 \text { or toxic for } \\
\text { reproduction category 2 together with toxic effects } \\
\text { on endocrine organs) }\end{array}$ \\
\hline EC Option 2 & $\begin{array}{l}\text { Substances which fulfill the WHO/IPCS (2002) } \\
\text { definition for an endocrine disruptor (i.e., external } \\
\text { substances causing adverse effects due to an } \\
\text { endocrine mediated mode of action) }\end{array}$ \\
\hline $\begin{array}{l}\text { EC Option 3- } \\
\text { Category 1 }\end{array}$ & $\begin{array}{l}\text { Endocrine Disruptor: Identical to option 2 } \\
\text { EC Option 3- }\end{array}$ \\
Category 2 & $\begin{array}{l}\text { Suspected endocrine disruptors: substances for } \\
\text { which the strength of evidence is not sufficient to }\end{array}$ \\
\hline EC Option 3- & $\begin{array}{l}\text { Endocrine active substances: substances which } \\
\text { have shown some endocrine activity but for which } \\
\text { there is not sufficient evidence to assign them in } \\
\text { category 1 or 2. }\end{array}$ \\
\hline EC Option 4 & $\begin{array}{l}\text { Based on the WHO/IPCS (2002) definition for an } \\
\text { endocrine disruptor and additionally considering } \\
\text { potency criteria. }\end{array}$ \\
\hline
\end{tabular}

a:not regarded in this screening as most of the example substances evaluated in this context are not classified for carcinogenicity or reproductive toxicity

severity, route, dose) was regarded in this screening study on natural substances.

The objective of this project was to assess the consequences of the EC proposals (options 2, 3, and 4) as well as the Industry Proposal for the identification and categorization of natural substances as endocrine disruptors.

The results of this project have to be regarded as results of a screening activity on basis of a limited data set assessing the impact of the different options to identify endocrine disruptors. It was not intended to prepare full monographs and final assessments of the endocrine disrupting potential of the example substances as required under different pieces of legislation. A final evaluation can only be achieved by a comprehensive assessment of all available data which was beyond the scope of this project. Conclusions presented here do not pre-empt regulatory conclusions eventually to be made under certain legislations.

\section{Methodology}

Genistein (CAS No. 446-72-0; isoflavone phytoestrogen), caffeine (CAS No. 58-08-2; alkaloid), vitamin D3 (CAS No. 67-970 ; pro-vitamin), and sucrose (CAS No. 57-50-1; disaccharide carbohydrate) have been selected for evaluation.

This screening activity focused on endocrine activities mediated via estrogen/androgen/thyroid/steroidogenesis
- EATS-modalities. This focus was selected, because these pathways are relatively well understood; most endocrine mediated effects known today are mediated by EATS modalities, and the OECD Guidance Document No. 150 on Standardised Test Guidelines for Evaluating Chemicals for Endocrine Disruption [2] provides consensus guidance on the interpretation of effects observed in studies performed according to OECD test guidelines.

The evaluation of the four substances is based on public available data. Literature searches were performed in the following databases: eChemPortal, which provided an overview on regulatory reports and monographs; PubMed and Toxline (bibliographic databases in the public domain); Science Citation Index (SciSearch) and Biosis (bibliographic databases in the non-public domain); internet searches to identify grey literature as well as database searches with specific relevance to ecotoxicity data like ECOTOX database and OECD QSAR toolbox.

Substance name and CAS number were used as search terms for searches in eChemPortal. Searches in bibliographic databases were performed by using a combination of the substance name and CAS number together with key words like endocrine, reproductive, repeated dose, fertility, developmental, mode of action. Regulatory reports and monographs obtained from eChemPortal were checked for data on repeated dose and reproductive toxicity as well as information on the mode of action. Hits from bibliographic databases were screened on abstract level to identify publications on repeated dose and reproductive toxicity or mode of action which were obtained in original and evaluated.

Substance name and CAS number were used as search terms for searches in the ECOTOX database and OECD QSAR toolbox. Search results were screened for hits identifying any studies according to guideline or similar to guideline as mentioned for wildlife in the OECD Guidance Document No. 150 , especially those for which guidance for interpretation of results has been provided like e.g., amphibian metamorphosis assay, fish sexual development test, or fish lifecycle toxicity test.

As it was not the intention of this project to document and discuss all available information on possible endocrine activities of a certain substance in a weight-of-evidence approach, a top-down approach has been chosen. Available in vivo guideline studies mentioned in OECD GD 150 [2] for the substances under evaluation were analysed (primary focus on studies for mammalian toxicity). If no respective guideline studies were available, other longer-term studies (repeated dose studies, reproductive toxicity studies) were evaluated. If several valid guideline studies were available for one substance, the one with the lowest effect level was selected as the key study. Epidemiological findings or reports on environmental organisms were only regarded if they were available in a summarized version.

Effects described in the publications were compared with the endpoints regarded as relevant for endocrine disruption modalities as described in the OECD guidance document No. 
150 to analyse if a substance elicits EATS-mediated effects or not.

The focus of this screening was on EC options 2 to 4 laid down in the EC roadmap [3] and beyond that on an Industry Proposal providing criteria for identification of endocrine disruptors [4] which are shortly summarized in Tables $\mathbf{1}$ and 2, respectively. EC option 1 was not regarded, as most of the example substances evaluated in this context are not classified for carcinogenicity or reproductive toxicity, so that the interim criteria of the biocide and pesticide regulation could not be applied.

Table 2. Industry Proposal presented by Cefic and ECPA for the identification of endocrine disruptors in a weight of evidence approach.

1. The substance reveals primary endocrine mediated adverse effects in an intact organism (mammals and/or nonmammals).

2. There is a causal relationship between the primary ED mode of action and the adverse effect.

3. Effects are observed after exposure via a relevant route.

4. Effects are observed at relevant doses.

5. Relevance to humans or to animal species living in the environment cannot be excluded.

6. Potency should be taken into consideration: The lead endocrine mediated adverse effect in mammals should be observed at doses equal to or below the dose threshold set out for substances to be classified as 'Category 1 Specific Target Organ Toxicity' following repeated exposures ("STOT-RE” Cat. 1) according to the requirements of Regulation (EC) No. $1272 / 2008$

7. Severity of effect should also be taken into consideration regarding the degree of difference from the untreated population either at the individual or population level.

8. Endocrine-mediated adverse effects should only be considered if they are the most sensitive/lead toxic effect of the substance.

All four options assessed are based on the WHO/IPCS Definition of an endocrine disruptor [5]:'An endocrine disruptor is an exogenous substance or mixture that alters function(s) of the endocrine system and consequently causes adverse health effects in an intact organism, or its progeny, or (sub) populations.' This definition determines the core elements of the screening: to assess

a) whether the substances of interest elicit adverse effects and

b) if these are plausibly linked to an endocrine mode of action.

Whereas the EC options 2 and 4 as well as the Industry Proposal do not differentiate between different categories of endocrine disruptors, EC option 3 distinguishes between Endocrine Disruptors (Cat. 1; i.e., identical to EC option 2), Suspected Endocrine Disruptors (Cat. 2) and Endocrine Active Substances (Cat. 3). Figure 1 shows the decision tree to be applied for the subcategorization of substances under EC option 3. This decision tree has been set up in a manner similar to the screening methodology used by JRC to facilitate the comparison of the results of both projects. The decision tree described in Figure 1 was developed on basis of preliminary results presented by JRC and published on the European Commission's Website [6], as the full report from JRC [1] was only available at the end of this project.

Analysis of adverse effects described in the substance specific studies was performed on basis of the OECD Guidance Document 150 [2]. According to the advice provided in Table 3.1 'Endpoints relevant for endocrine disruption modalities in Test Guidelines and other endocrine disruption-sensitive assays' of OECD Guidance Document 150 adverse effects were assigned as 'EATS-diagnostic effects,' 'potentially EATS-mediated effects', or non-EATS mediated effects. 'EATS-diagnostic effects' are all endpoints which are possibly related to (anti-)estrogen, (anti-)androgen, thyroid or steroidogenesis mediated effects, for example effects on the weight of thyroid or reproductive organs, histology of thyroid or reproductive organs, sperm parameters, estrous cyclicity, age at pubertal separation, age at vaginal opening, anogenital distance.

EATS-diagnostic effects (=endpoints for EATS-mediated activity) have to be clearly distinguished from 'endpoints potentially sensitive to, but not diagnostic of, EATS modalities' (also referred to as 'potentially EATS-mediated effects'), like effects on the weight of adrenals or pituitary, histopathology of adrenals or mammary glands, male and female fertility, gestation length, number of implantations or corpora lutea, litter size.

To clarify if the observed adverse effects could be plausibly linked to an endocrine mode of action additional, in vitro and/ or in vivo information on the mode of action were collected and all available information on adverse effects and mode of action was analysed with respect to the possible consequences for the classification. For the sake of this screening activity a plausible link was assumed in case available mechanistic data support the possible mechanism discussed for a certain effect, e.g., if effects on sperm could be related to (anti-)estrogenic or (anti-) androgenic effects. If there is mechanistic evidence that the substance reveals e.g., anti-androgenic effects a plausible link was assumed without further examination of the likelihood that this MOA has caused the observed averse effect.

Depending on the different options to be discussed, additional aspects have to be regarded besides these core elements: e.g., potency, lead toxic effect, severity, relevant route of exposure and relevant dose.

For potency considerations the LOAEL for the (potentially) EATS-mediated adverse effect was compared with guidance values (cut-offs) set in Regulation (EC) No. 1272/2008 for classification of Specific Target Organ Toxicity-Repeated Exposure (STOT-RE) as recommended by BfR [16] and in the Industry Proposal. If the LOAEL was equal or below the guidance value for STOT-RE Category 1, the substance was classified as endocrine disruptor under the criteria of EC Option 4. If the LOAEL 


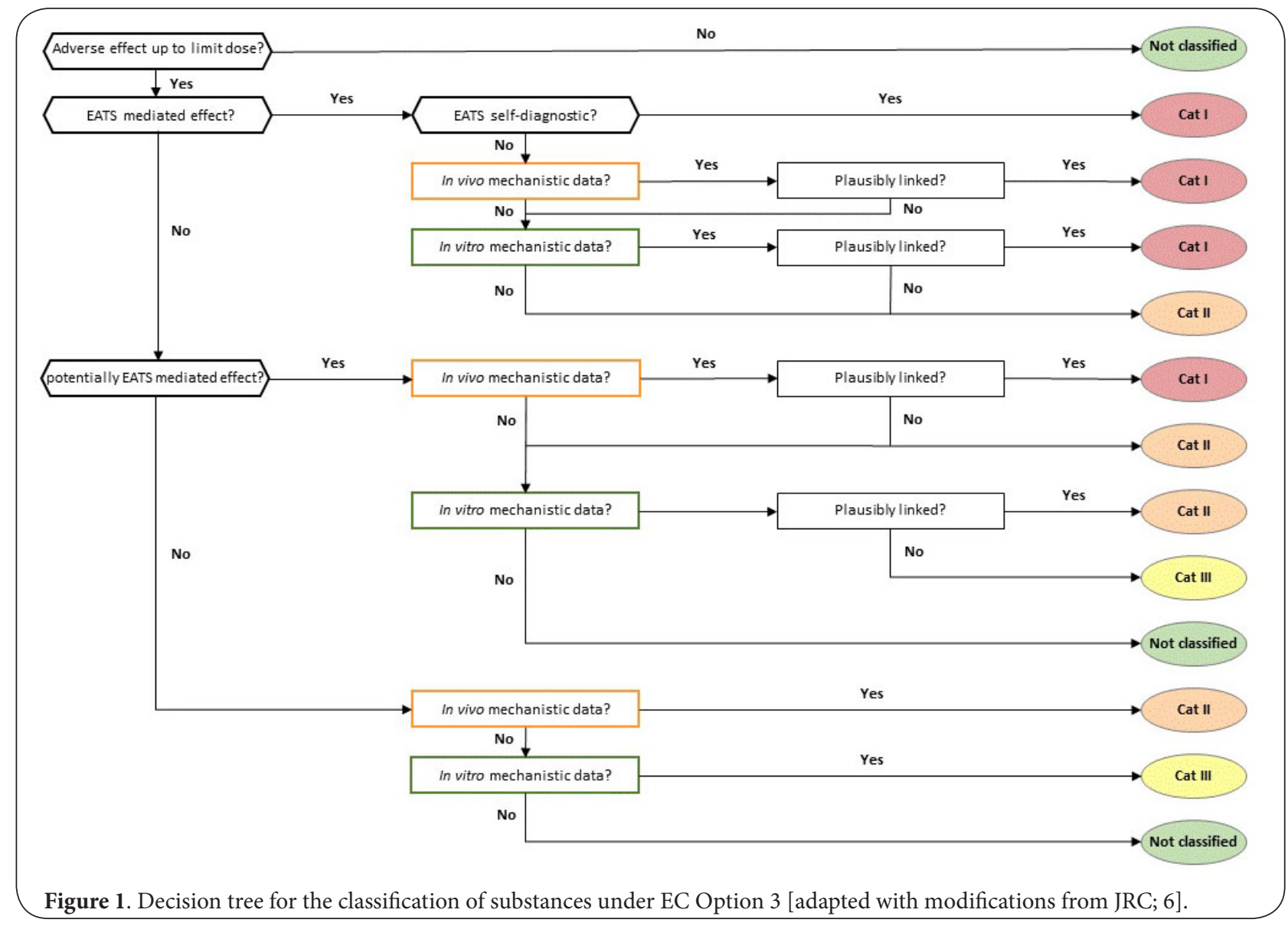

Table 3. Result of endocrine disruptor classification in relation to the different options under discussion on basis of EATS related effects.

\begin{tabular}{|c|c|c|c|c|}
\hline Substance & EU-Option 2 & EU-Option 3 & EU-Option 4 & $\begin{array}{l}\text { Industry } \\
\text { Proposal }\end{array}$ \\
\hline Sucrose & Not classified & Not classified & Not classified & Not classified \\
\hline Genistein & $\begin{array}{l}\text { Endocrine } \\
\text { Disruptor }\end{array}$ & $\begin{array}{l}\text { Endocrine } \\
\text { Disruptor Cat. } 1\end{array}$ & $\begin{array}{l}\text { Endocrine } \\
\text { Disruptor }\end{array}$ & $\begin{array}{l}\text { Endocrine } \\
\text { Disruptor }\end{array}$ \\
\hline Caffeine & $\begin{array}{l}\text { Endocrine } \\
\text { Disruptor }\end{array}$ & $\begin{array}{l}\text { Endocrine } \\
\text { Disruptor Cat. } 1\end{array}$ & Not classified & Not classified \\
\hline Vitamin D3 & Not classified & $\begin{array}{l}\text { Endocrine Active } \\
\text { Substance Cat. } 3 \\
\star\end{array}$ & Not classified & Not classified \\
\hline
\end{tabular}

* classification due to single in vitro findings on influences on aromatase activity

was higher than the guidance value for STOT-RE Category 1 no classification resulted under the criteria of EC Option 4.

For considerations on lead toxic effect the NOAEL of the endocrine mediated adverse effect was compared to the NOAEL of non-EATS mediated effects. The lowest NOAEL determined the lead toxic effect.

Currently, clear defined criteria for severity of the effect are missing. This discussion was performed on a case-by-case basis.

A relevant route of exposure was assumed if the applica- tion route was typical for the general use of the substance, e.g., oral application for substances used as food additives. Doses were regarded as relevant if they did not exceed the limit dose of the test guideline or the maximum tolerable dose of the substance, i.e., did not cause excessive toxicity.

\section{Results}

There are only very limited data available for sucrose. No guideline studies on mammalian or non-mammalian repro- 
ductive or repeated dose toxicity as recommended in OECD Guidance Document 150 could be identified in the published literature. Investigations on possible carcinogenic activity of sucrose as well as some limited investigations on developmental toxicity as summarized in ACGIH [7] do not indicate any EATS mediated adverse effects. Sucrose was not active in two in vitro assay systems: the hERTa assay for estrogen receptor transcriptional activation (sucrose tested at concentrations of $1 \mathrm{E}-4$ to $1 \mathrm{E}-10 \mathrm{M}$ ) and the $\mathrm{H} 295 \mathrm{R}$ steroidogenesis assay (at $62.5-250 \mu \mathrm{M}$ ). Because of the negative outcome in these in vitro assays, sucrose has not been further tested in in vivo assays in this study [8]. No studies regarding relevant ecotoxic effects ("Wildlife Screens and Tests" according to OECD Guidance document 150 or similar studies on endocrine disruption) could be identified for sucrose. Based on these limited data it is concluded that sucrose would not be classified as an endocrine disruptor when focusing on EATS mediated effects, independently of the option applied, as no EATS related endocrine effects have been identified in the available studies. Effects on insulin sensitivity are addressed in the discussion section.

Genistein, a phytoestrogen, is a substance with a very broad database. Reproductive toxicity and endocrine activity of the substance has intensively been investigated and reviewed by NTP [9]. A multi-generational reproductive toxicology study in Sprague-Dawley rats is regarded as most relevant with respect to endocrine activity regarding the effects and effect levels observed [10]. Rats were exposed to 0, 5, 100, $500 \mathrm{ppm}$ genistein in food (ca. $0,0.3,7$, or $35 \mathrm{mg}$ genistein $/ \mathrm{mg}$ genistein $/(\mathrm{kg} \mathrm{bw}$ $x \mathrm{~d}$ ) for males and $0,0.5,10$, or $51 \mathrm{mg} /(\mathrm{kg} \mathrm{bw} x \mathrm{~d}$ ) for females). A number of EATS-diagnostic effects were observed in rats (for summary, see Table 4), which might be due to different modes of action (MoA):

1. mammary gland hyperplasia in males: indicative of estrogen agonistic effect. Moreover, mammary gland hyperplasia in the male rat has been assessed as one of the most sensitive markers of estrogenic endocrine disruption [11].

2. accelerated vaginal opening: indicative of estrogen agonistic effect, maybe also mediated by estrogen-antagonistic, androgen-agonistic and androgen-antagonistic as well as other steroidogenesis related effects.

3. AGD decreased: indicative of androgen-antagonistic effect in males, but effects in males and females may be also mediated by estrogen-agonistic, estrogen-antagonistic and androgen-agonistic effects.

4. estrous cycle changed: maybe mediated by estrogenagonistic, estrogen-antagonistic, androgen-agonistic and androgen-antagonistic effects.

According to OECD Guidance Document 150 it is not possible to exactly assign the individual effects to a specific MoA, as they are listed under more than one EATS -mediated effect. The findings from the multi-generational study are supported by similar findings from other in vivo studies which described comparable effects on e.g., AGD and mammary glands [e.g.,
12-14]. The endocrine mediated effects observed after genistein exposure indicate that the postnatal development and sexual maturation of the animals are affected. As this might impair the physiological function these effects are regarded as adverse.

In vivo investigations on the MoA provide evidence for estrogenic activity of genistein, which is weak in comparison to the natural hormone [9]. Data from in vitro measurements of estrogenicity consistently demonstrate a weak estrogenic activity of genistein, with potencies well below that of estradiol [9]. In more recent studies, it was demonstrated that genistein acts as agonist and both activates ER-alpha- and ER-beta- estrogen response element [15].

Altogether, observed adverse effects point to an EATSmediated MoA. Estrogenic activity might be one explanation for the observed effects. In vivo and in vitro studies on the possible MoA also point to an estrogenic activity of genistein. For the sake of this screening activity it can reasonably be assumed that the adverse effects are mediated by an endocrine mode of action. Therefore, genistein would be classified as ED if the criteria of EC Option 2 and 3 are applied.

For the classification of a substance as 'STOT-RE Cat 1', a guidance value ('cut-off') of $\leq 10 \mathrm{mg} /(\mathrm{kg}$ bw $x \mathrm{~d}$ ) as a LOAELoral in a 2-generation study has been proposed [16]. In the multigenerational NTP-study, a LOAEL of $7 \mathrm{mg} /(\mathrm{kg}$ bw $\mathrm{x}$ d) was determined for mammary gland hyperplasia in males. The LOAEL of $10 \mathrm{mg} /(\mathrm{kg}$ bw $x \mathrm{~d})$ for decreased AGD in F3 females also meets this criterion.

Additionally, there is a LOAEL of $2.5 \mathrm{mg} /(\mathrm{kg} \mathrm{bw} \times \mathrm{d})$ for Leydig cell hyperplasia and slight decrease in sperm count in a subacute oral toxicity study in male mice [17]. Such an effect may also be due to endocrine disruption (see OECD Guidance document 150 on TG 407) of a substance. According to the Joint DE-UK Position Paper [16], a LOAELoral of $\leq 30 \mathrm{mg} /(\mathrm{kg}$ bw $x$ d) has been proposed as a 'cut-off' for 'STOT-RE Cat 1'for subacute toxicity studies.

As the LOAEL for genistein from a multi-generational study, and the LOAEL from a subacute study are equal or below the STOT-RE Cat 1 cut-off value the potency criterion is met and genistein would be classified as ED if the criteria of EC Option 4 are applied.

Genistein would also be classified as endocrine disruptor if the criteria of the Industry Proposal would be applied as the additional criteria mentioned in the Industry Proposal are also met:

1. Effects were observed after application via a relevant route of exposure (oral), especially in light of the observation that people are usually exposed by ingestion of isoflavones either in their food or in dietary supplements.

2. Exposure was towards relevant doses, i.e., doses which did neither exceed the maximum tolerable dose nor the limit of guideline recommendations.

3. Relevance to humans has to be assumed as effects were observed in different species and there is no evidence 
Schuhmacher-Wolz et al., Journal of Toxicology and Health 2017,

http://www.hoajonline.com/journals/pdf/2056-3779-4-2.pdf

doi: $10.7243 / 2056-3779-4-2$

Table 4. Adverse effects observed in the multi-generation study with Sprague Dawley rats which received genistein in the food [10].

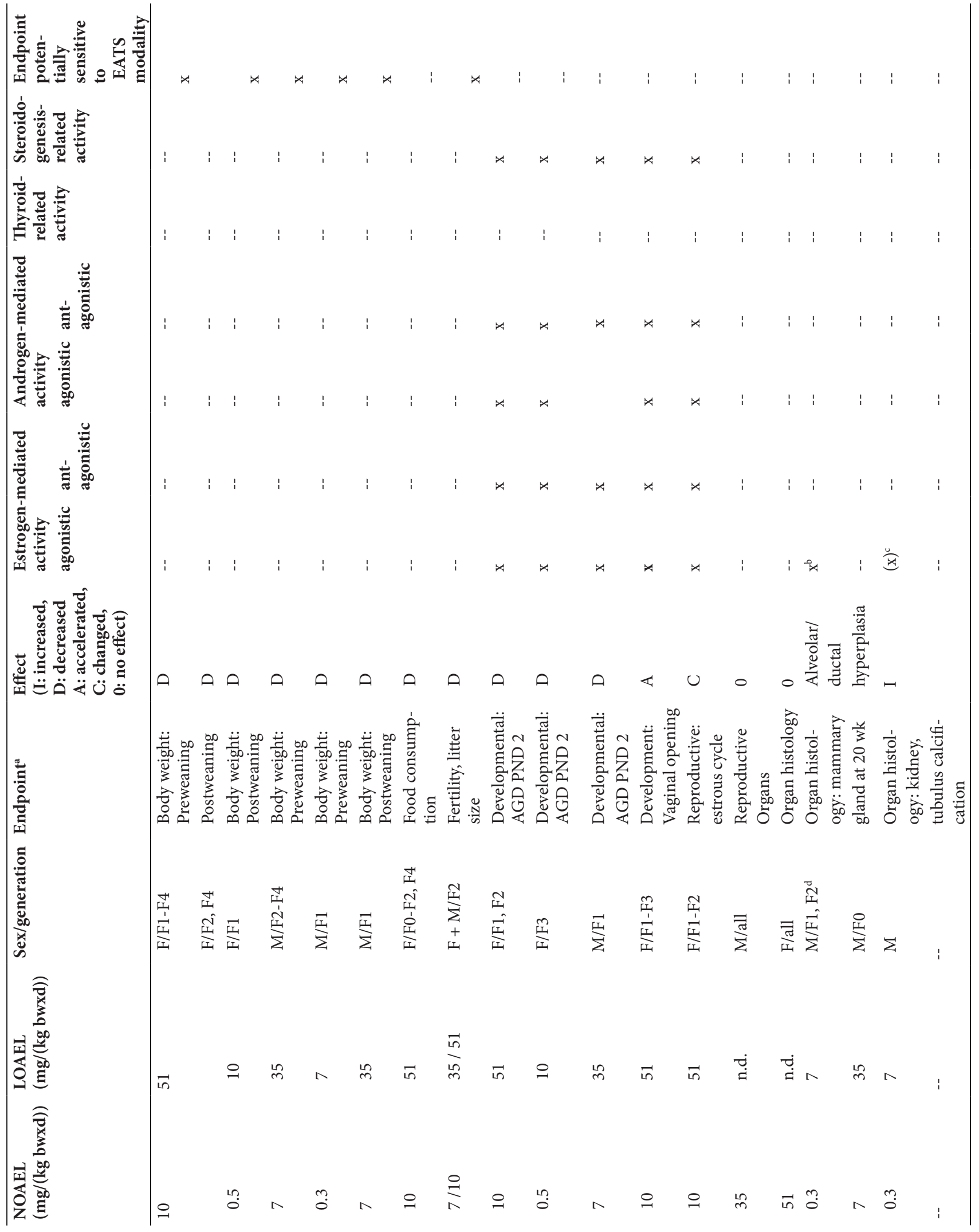

*: Data from summary table; a: significant changes relative to control; b: Not mentioned in OECD GD 150 [2] for EATS endpoints in TG 416, but for TG 408, TG 451-3, TG 443 and 407. Also, evaluation of NTP-studies concluded that mammary gland hyperplasia in the male rat is one of the most sensitive markers of estrogenic endocrine disruption [11]; c: possibly related to estrogenic activity [10]; d: a significant positive trend was observed in F1, F2, and F3. 
of species-specific mechanisms. Rather, the underlying mechanisms are widely distributed and conserved between different species.

4. Final evaluation of the severity of the effects is not possible at the moment due to missing criteria for severity. Taking into account the discussion of the EFSA opinion on the hazard assessment of endocrine disruptors, one aspect would be the reversibility of an effect. From the existing studies it is not known whether the observed effects are reversible or not.

In summary, on basis of the evaluated data in mammals it is concluded that genistein would be classified as an endocrine disruptor independently of the option applied as genistein causes EATS-diagnostic effects which can plausibly be linked to an endocrine mode of action. Further, the potency criteria as well as the additional criteria of the Industry Proposal are met.

The available data on ecotoxicity are not sufficient for an evaluation or classification of genistein as an endocrine disruptor, especially as a life-cycle test with zebra fish is not published and information is only available from secondary sources [18]. But the available information are in line with the observations from mammal studies: According to [2], an effect on fertilization as observed in the fish life cycle test per se may indicate potentially EATS mediated effects but is not EATS-diagnostic. Further investigations in zebra fish confirm an estrogenic activity of genistein [19]. Other investigations point only to limited effects of genistein on reproductive parameters and hormone levels in fish [20-22].

Caffeine is an alkaloid found in various plant constituents and has a long history of human consumption, especially in tea and coffee. It is also an ingredient added to a variety of foods, e.g., baked goods, ice creams, soft candy, cola-type beverages and so-called "energy drinks". Some medicines and cosmetics also contain caffeine [23].

Toxicity after repeated exposure as well as reproductive toxicity have been intensively investigated. Substance data are reviewed e.g., by the International Agency for Research on Cancer [24] and the OECD-SIDS on caffeine [25]. A reproductive toxicity study of caffeine with rats using the Reproductive Assessment by Continuous Breeding (RACB) protocol was regarded most relevant for this screening activity [26]. In this study rats were gavaged with $0,12.5,25$, and $50 \mathrm{mg}$ caffeine/ ( $\mathrm{kg}$ bw $\mathrm{xd}$ ). During 16 weeks of cohabitation, live pup weight adjusted for litter size was decreased by 7,7 , and $8 \%$ in the $12.5,25$, and $50 \mathrm{mg} /(\mathrm{kg}$ bw $x \mathrm{~d}$ ) dose groups, respectively. This effect is potentially sensitive to, but not diagnostic of, EATS effects. No differences were observed in other reproductive endpoints (litters per pair, number of live F1 pups per litter, proportion of pups born alive and sex ratio).

The decreased pup weight occurred concomitant with a dose-dependent reduction of weight gain in dams. Throughout the study, the body weights were less than controls in the F0 males and females by 4-19\% [26]. At necropsy, many relative organ weights were increased in all dosed groups, these differences were attributed to the decrease in terminal body weights. No treatment-related gross or microscopic lesions were observed in kidneys, testes or epididymis of F0 males [26]. Similar findings were also observed in the second generation evaluation (Task 4 of the RACB).

Evaluation of computer-assisted sperm analysis data revealed a slight treatment-related decrease (3\%) in percent motile sperm in the $50 \mathrm{mg} /(\mathrm{kg}$ bw $x \mathrm{~d}$ ) F0 males compared to controls. The sperm velocity was decreased (7-12\%) in all treated males. The average sperm radius was also decreased (22-36\%) in males of all dose groups, and the percent normal sperm was decreased slightly in the $50 \mathrm{mg} /(\mathrm{kg} \mathrm{bw} \mathrm{xd}$ ) males. Similar effects on sperm parameters were observed in F1 males.

Effects on sperm parameters and on male reproductive organs (testis and accessory organs weight or histology) have also been observed in other, non-guidelines studies on reproductive toxicity or in studies on effects on male sex organs. Some studies have also described alterations in serum levels of sex hormones after oral exposure of animals to caffeine. Generally, these effects occurred at doses causing marked general toxicity such as reduced weight gain or terminal body weight. At lower doses used in subchronic oral toxicity NTPstudies with rats and mice (comparable to OECD TG 408), no such effects have been described (sperm parameters were not investigated in these studies).

The effects observed in sperm are EATS-diagnostic and indicative of estrogen- or androgen-mediated agonistic/ antagonistic activity as well as other steroidogenesis related activity (see Table 5). However, they could not clearly be attributed to a certain MoA.

The pharmacology of caffeine has been extensively studied. The effects of caffeine are predominantly related to its antagonistic activity at adenosine receptors. Caffeine is also known as a non-specific phosphodiesterase inhibitor, leading to an increase in cyclic nucleotides [23]. With respect to EATSmediated effects, the results of in vitro and in vivo indicate that caffeine does not bind to the estrogen receptor in vitro, but may affect steroidogenesis in general. Results from in vivo guideline assay with immature [8] and mature male rats $[27,28]$ also point to an effect of caffeine on steroidogenesis. More specifically, the data suggest that caffeine may express anti-androgenic activity in male rats. An assay in immature female rats also is in accordance with an effect on steroidogenesis [8]. Unfortunately, estradiol could not be measured in that assay; therefore, no conclusions can be drawn regarding effects on estrogen levels. In a rat study following the RACB protocol the most important effects with respect to endocrine effects were alterations of sperm parameters, also pointing to an effect of caffeine on steroidogenesis. The effects on sperm were not severe, but are considered adverse according to criteria of the US EPA [29]. The mechanistic investigations support the interpretation that the adverse effects observed in sperm may be mediated by endocrine effects.

Altogether, it can be stated that caffeine may induce EATS- 
Table 5. Adverse effects observed in the RACB study with caffeine with Sprague Dawley rats [26].

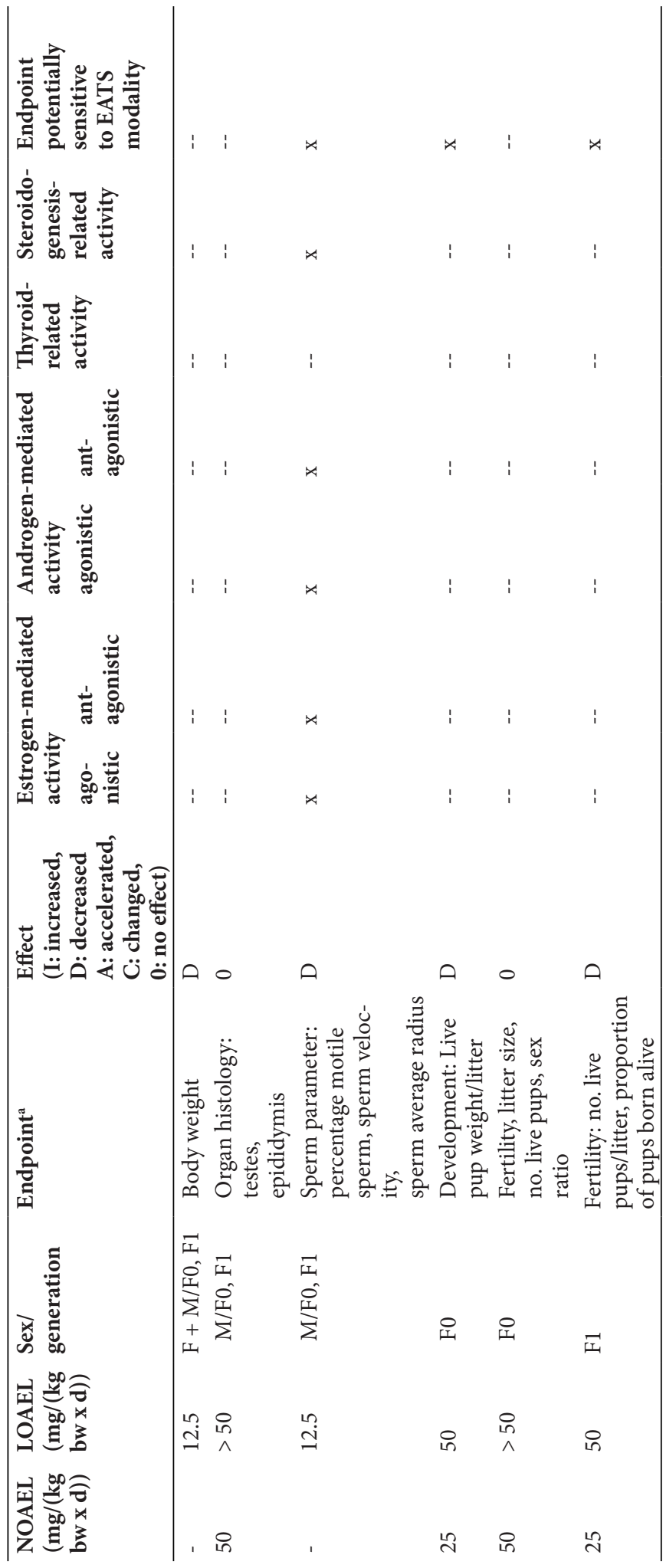

a:significant changes relative to control 
diagnostic effects on sperm at doses which already cause general toxicity. Whether the EATS-diagnostic effects are secondary to or independent from these general toxic effects is unclear and could not be clarified within this screening activity. For the sake of this screening and taking into account the information on the MoA it is assumed that sperm effects may be caused by an endocrine mode of action. Therefore, caffeine would be classified as ED or ED Category 1 if the criteria of EC Option 2 or 3 are applied, respectively.

A LOAEL of $12.5 \mathrm{mg} /(\mathrm{kg} \mathrm{bw} \times \mathrm{d})$ was obtained in the RACB study for sperm effects. Thus, the LOAEL for EATS-mediated effects is above the cut-off value for proposed for classification of a substance as 'STOT-RE Cat 1' of $\leq 10 \mathrm{mg} /(\mathrm{kg}$ bw $x \mathrm{~d})$ in a two-generation study. Therefore, the potency criterion is not met and no classification according to EC option 4 would result. Caffeine would also not be classified as endocrine disruptor if the criteria of the Industry Proposal would be applied as some of the additional criteria mentioned in the Industry Proposal are not met:

1. As outlined above caffeine induces adverse effects, which may plausibly be linked with an EATS-mediated mode of action.

2. Effects were observed after application via a relevant route of exposure (oral), especially in light of the observation that people ingest caffeine in drinks or food.

3. Exposure was towards relevant doses, i.e., doses which did neither exceed the maximum tolerable dose nor the limit of guideline recommendations. However, adverse effects occurred at doses leading to general toxicity.

4. Relevance to humans may principally be assumed as effects were observed in different species and there is no evidence of species-specific mechanisms. However, from the large epidemiological database EFSA [23] has concluded that 'no health concerns in relation to ... male fertility have been raised by other bodies in previous as sessments for this level of habitual caffeine consumption and no new data have become available on these or other clinical outcomes which could justify modifying these conclusions'.

5. With respect to potency, effects on sperm parameters were observed at $12.5 \mathrm{mg} /(\mathrm{kg}$ bw $\times \mathrm{d}$ ) (LOAEL) caffeine not fulfilling the criteria for the classification of STOT RE Cat. 1.

6. Final evaluation of the severity of the effects is not possible at the moment due to missing criteria for severity. Taking into account the discussion of the EFSA opinion on the hazard assessment of endocrine disruptors one aspect would be the reversibility of an effect. Some studies indicate that the effects may be long-lasting, but there are no data available indicating a permanent irreversible effect on sperm parameters or reproductive effects.

7. The effects on sperm parameters (and other outcomes which may also be sensitive, but not diagnostic for EATSmediated effects) are observed at doses which also lead to general toxic effects (reduced body weight gain).

Therefore, they are not considered the lead toxic effect. In summary, on basis of the evaluated data in mammals it is concluded that caffeine would only be classified as an endocrine disruptor under EC option 2 (ED) and EC option 3 (ED Category 1). No classification would result under EC option 4 and the Industrial Proposal as a) caffeine does not fulfil the potency criterion and b) EATS-mediated effects are not the lead toxic effects.

No available data in non-mammals were identified which were considered relevant within the scope of this evaluation.

Vitamin D3 (Vit-D3) is a pro-vitamin, which is produced in the human body in the presence of sunlight and also added from exogenous sources. Vit-D3 is an essential compound, both too low (hypovitaminosis) and too high levels (hypervitaminosis) are associated with serious health effects. There is a large body of literature dealing with effects of Vit-D3 undersupply and oversupply. Due to its physiological role repeated application of high doses of Vit-D3 results in effects on body weight, increased blood calcium levels (hypercalcae$\mathrm{mia}$ ), hypercalciuria and soft tissue calcification [30-33]. These effects have been observed in animals and in humans [34]. None of these effects are diagnostic for EATS or potentially sensitive to EATS modalities. Investigations on the possible developmental effects of Vit-D3 have been performed with its active metabolite 1,25-dihydroxyvitamin D3 [e.g., 35]. Rat studies did not reveal any adverse effect which might be EATSmediated. In the rabbit study effects potentially sensitive to EATS modulation like reduction of litter size and decreased survival of viable pups were only observed in the high dose group which already caused mortality. These effects on pups are probably secondary to maternal toxicity and are not used for further Vit-D3 evaluation.

The mechanism of Vit-D3 and its interaction with the parathormone system is very well investigated and understood, both in vivo and in vitro [e.g., 34,36]. Besides the interaction with the parathormone system the Vit-D3 metabolite 1,25-Dihydroxyvitamin D3 has been shown to act as selective aromatase modulator, decreasing aromatase expression in breast cancer cells and the breast adipose tissue surrounding a breast tumour, resulting in a reduction of the local production of estrogens within the breast. Additionally, 1,25-dihydroxyvitamin D3 also represses estrogen receptor expression in breast cancer cells in vitro, two mechanisms possibly relevant for breast cancer therapy [37]. For the sake of this screening activity this publication was taken as in vitro evidence for an EATS-mediated MoA.

No data on Vit-D3 and ecotoxicity covering the endpoints mentioned in the OECD guidance document 150 and within the scope of this project could be identified in the literature search.

In summary, on basis of the evaluated data in mammals and with the focus on EATS-mediated effects it is concluded that Vit-D3 would not be classified as an endocrine disruptor 
if the criteria of EC option 2, option 4 and the Industry Proposal are applied, as there is no evidence that Vit-D3 causes EATS-mediated adverse effects. Consequences for effects of Vit-D3 on the parathormone system and the consequences for a possible classification as ED are addressed in the discussion section.

A special feature arises on basis of the in vitro data pointing to a MoA which interacts with the EATS system. Due to in vitro mechanistic data obtained with the active metabolite 1,25-dihydroxyvitamin D3 in absence of EATS-diagnostic or potentially EATS-mediated adverse effects but presence of data on general toxicity (body weight loss) in rat studies these in vitro mechanistic data formally would result in a classification of Vit-D3 as Cat. 3 substance, if the criteria of EC option 3 would be applied. It means that under this criteria Vitamin D3 would be recognized as substance which has shown some endocrine activity but for which there is not sufficient evidence to assign it in category 1 or 2 . It has to be recognized that the quality and reliability of these in vitro results has not been checked within this screening approach. Anyway, the reported results were used to demonstrate the consequences of such a database in the evaluation of a possible endocrine activity of a substance.

\section{Discussion}

The results of this project have to be regarded as the outcome of a screening activity on basis of a limited data set assessing the impact of the different options to identify endocrine disruptors. It was not intended to prepare full monographs and final assessments of the endocrine disrupting potential of the example substances as required under different pieces of legislation. A final evaluation can only be reached within a much more comprehensive weight of evidence approach considering all available data which would go beyond the scope of this project. Conclusions drawn do not pre-empt regulatory conclusions eventually to be made under these legislations. But working up these examples made obvious that even for a screening activity, an in-depth discussion of in vivo evidence and mechanistic data is required to be able to compare the evidence with the categorization criteria.

All four options for classification of ED included in this screening study are based on the WHO/IPCS definition for endocrine disruptors, i.e., the core element of classification is the identification of an adverse effect due to an alteration of the function of the endocrine system. Recognition of such a possibly endocrine mediated adverse effect is the first step of the evaluation. The OECD Guidance Document on Standardized Test Guidelines for Evaluating Chemicals for Endocrine Disruption [2] is very helpful in identifying EATS mediated effects. But, further guidance for identification of other than EATS mediated effects is currently missing and hampers the identification of such effects. That was the reason for focusing this screening activity on EATS mediated effects. But for future substance evaluations it is considered necessary to have further guidance for other endocrine mediated effects available to prevent that such effects are overlooked as discussed below.

The four example substances are substances of our daily live which we consume e.g., with our food, as drugs or dietary supplement and for which a safe use has been proven. The example substances reached different results in this screening.

1. Substance not to be classified as ED under any option (sucrose),

2. Substance to be classified as ED independently of the option applied (genistein),

3. Substance to be classified as ED only under certain but not all options (caffeine),

4. Substance predominantly not to be classified as ED with respect to EATS mediated effects, however interacting with other hormonal systems (Vit-D3).

Genistein is a substance with a broad and consistent database which resulted in the classification as ED independently of the option applied. Nevertheless, the result of this screening activity should not call the safe use of this substance as a food supplement into question. For example the EFSA Panel on Food Additives and Nutrient Sources added to Food (ANS) concluded that there is no association between the intake of isoflavones from food supplements and harmful effects on mammary gland, uterus and thyroid in peri- and postmenopausal women [38].

Caffeine is also a substance with an extensive database. However, the data are less clear cut than for genistein. Caffeine causes general, unspecific toxicity (changes in body weight) in the same dose range at which EATS-diagnostic effects (effects on sperm parameter) occur. For this screening it was assumed that the EATS-mediated effects are independent from general toxicity. However, this example reveals that it is necessary to address and clarify in a final substance evaluation the interrelation between endocrine mediated and other toxic effects. It could be discussed how far the marginal albeit statistical significant effects on sperm should be regarded as adverse or only as biological variability. Epidemiological data indicate no risk for effects on male fertility by habitual coffee consumption [23]. In a conservative approach and following the recommendation of the US EPA [29], the statistical significant effects on sperm have been regarded as adverse for this screening. For future evaluations it might be a matter of debate which effects should be regarded as adverse or biological variability. Available mechanistic investigations for caffeine point to a steroidogenic activity of caffeine. As effects on sperm parameters may be mediated by several mechanisms (anti-/estrogenic or anti/-androgenic or steroidogenesis related) a plausible link between adverse effect and endocrine MoA has been assumed for the sake of this screening. This points to the difficulties evaluators have to overcome in the future: Investigations on adverse effects and MoA are often performed in different studies. At the same time certain hormonal effects may be mediated by several modes of action which makes it difficult to reach a clear-cut decision if there 
is a plausible link between the adverse effect and the MoA. At the same time the current proposals for the identification of endocrine disruptors under biocide and pesticide regulation require clarification if the adverse effect of interest is a consequence of the endocrine mode of action. If this can be unequivocally demonstrated for the majority of substances to be evaluated in the future remains questionable.

Vit-D3 has not been classified as an endocrine disruptor under the EC option 2, 4, or the Industrial Proposal focusing on EATS-mediated effects. However, due to single in vitro mechanistic data obtained with the active metabolite 1,25-dihydroxyvitamin D3 in absence of EATS-diagnostic or potentially EATS-mediated adverse effects but presence of data on general toxicity (body weight loss) a classification of Vit-D3 as Cat. 3 i.e., as endocrine active substance would result, if the criteria of EC option 3 would be applied. This example reveals that a) a single in vitro assay may have far-reaching consequences and b) classification of a substance only on basis of in vitro assays together with some evidence of general toxicity in experimental animals seems to be a questionable procedure. It should be discussed which role single in vitro findings should play in future discussions on classification. This example very nicely demonstrates that application of formal criteria without overall weight of evidence assessment might result in misinterpretations. The effects on aromatase activity expression have been observed in breast cancer cells in vitro $[37,39,40]$ and in immunocompromised mice bearing MCF-7 xenografts [41]. The latter study was not regarded for the classification due to the artificial situation generated in mice. The data indicate that the effects on aromatase seem to be restricted to tumorigenic cells/tissues (no such effects were observed in ovaries and uteri) and in no way associated with the body weight loss observed in other in vivo studies.

Beyond the scope of this screening activity it has to be recognized that Vit-D3 would be classified as an endocrine disruptor with high potency in the microgram per kilogram body weight range if other (i.e., non EATS mediated) hormonal systems are regarded, because - if given in excess - Vit-D3 disrupts the regulatory circuit of calcium homeostasis including parathyroid hormone. This underlines the importance that future substance evaluations should not only be restricted to EATS-mediated effects. But this also points to a major challenge for the future to derive guidance on the evaluation of reproductive effects similar to the way performed for EATSmediated effects in the OECD Guidance Document 150 [2]. Vit-D3 or its active metabolite cholecalciferol do not fulfill the WHO/IPCS definition of an endocrine disruptor in a strict sense, because they are natural substances physiologically present in the mammalian body which act as (pro-) vitamin and are essential for normal function. At the same time they are exogenous substances used e.g., as drugs or food supplements. Depletion as well as excess will disturb the normal function of the body with severe health problems, and therefore those substances are essential to life. This example underlines the importance of the restriction of the WHO/IPCS definition of an ED to exogenous substances. This recommendation should be strictly followed, even if endogenous substances are partially supplied externally for example in food supplements.

For sucrose no formal assessment on the basis of guideline studies or studies similar to guideline could be performed as no such studies investigating effects relevant for this screening were available. The limited toxicity and mechanistic data do not indicate any concern for EATS-mediated endocrine disruptive effects. Further, the long lasting experience on the consumption of sucrose does not point to any EATS mediated effects in humans. Consequently, it has been concluded that sucrose would not be classified under any option evaluated. Animal studies, mostly with rodents, have provided evidence that high-sucrose diets can decrease insulin sensitivity, i.e., interact with another - non EATS - hormonal system. In humans, a similarly clear relationship is missing [42]. A systematic review of human intervention studies in healthy adults on the effects of sucrose on metabolic health provided little evidence for significant effects on plasma glucose or insulin and no evidence of any consistent difference between sucrose and starch or other carbohydrates in respect of the basic markers of insulin sensitivity [43]. Also, based on the evaluation of the FDA GRAS committee [44] it can be assumed that sucrose does not cause disruption of the insulin system which regulates the glucose levels in the body, i.e., sucrose would probably not be classified even if other endocrine systems beside EATS would be regarded. The outcome of the evaluation within this study is in good agreement with the results of the JRC screening study which also did not identify sucrose as an endocrine disruptor under any option. Moreover, the Standing Committee on Plants, Animals, Food and Feed of the European Union concluded in its review report for the basic substance sucrose that "Sucrose is not a substance of concern and does not have an inherent capacity to cause endocrine disrupting (according to the interim criteria in Regulation 1107/2009), neurotoxic or immune-toxic effects" [45].

All assessments presented here are based on data on nonhuman mammalian toxicity. Epidemiological findings, which have been screened, but not evaluated in detail, are in good agreement with the data in experimental animals for sucrose, genistein and Vit-D3. However, for caffeine, the epidemiological data do not indicate a risk of endocrine disruption during habitual coffee consumption.

For none of the four substances under evaluation are guideline studies or non-guideline studies available addressing relevant endpoints in non-mammal vertebrates, indicating that there are still - sometimes relevant - knowledge gaps. For sucrose, caffeine and Vit-D3 no relevant non-mammal studies could be identified at all. Only for genistein a full life cycle test in fish has been identified. In the presentation of the results of this life-cycle study in secondary literature an effect on fertilization rate is described. Effects on fertilization rate are potentially sensitive but not diagnostic of EATS 
modalities, i.e., these findings in fish are not contradictory to the mammalian data.

EC option 4 as well as the Industry Proposal include potency considerations. According to the recommendation of the Joint DE-UK-Position Paper $[46,47]$ substances, which elicit endocrine disrupting activity at doses similar or below the doses relevant for STOT-RE Cat. 1 classification, should be classified as endocrine disruptors. But, it remains unclear what the consequences are for substances not fulfilling STOT-RE Cat. 1 but STOT-RE Cat. 2 criteria. Currently, there are no criteria available for potency considerations for data in non-mammals, meaning that EC option 4 and the Industry Proposal could not be adequately considered for non-mammal data. Discussion in a workshop hosted by the German Federal Institute for Risk Assessment) [47] and a recent publication from Slama et al. [48] indicate that potency criteria are not considered relevant for future identification of endocrine disruptors. However, it should be emphasized that-similar to the procedure for skin sensitizing substances -potency considerations might be helpful for the evaluation of endocrine active substances as potency aspects might help to range effects observed in relation to the potency of natural hormones. It can reasonably be assumed that endocrine active substances of weak potency (several magnitudes lower potency than physiologic hormones) will probably not disturb the physiologic system although such substances might elicit effects at higher doses tested in experimental animals.

Application of the Industry Proposals investigated in this screening is partially hampered by the fact that not all criteria listed are clearly defined. There remains space for interpretation (e.g., adequate dose, severity of effect), which might result in discordant interpretations. If this proposal should be applied in the future a more detailed guidance on the application of the single criteria would be required. Further, it is a matter of discussion what would be the consequences if one or two criteria out of the list of three additional criteria (potency, severity, lead toxic effect) for the identification of ED is/are not fulfilled. Should only substances be classified for which all criteria are fulfilled? Are the different criteria equal or would a ranking for the different criteria be necessary? For the example substance caffeine two of the three criteria (potency, lead toxic effect) were not fulfilled. Severity of the effect was also a matter of discussion (see discussion above on the evaluation of sperm parameters). Therefore, it was concluded that caffeine would not be classified if the Industry Proposal would be applied.

Currently, there are no detailed information requirements addressing the minimum data needed for investigating possible endocrine activity of a substance. This might influence the identification of ED under certain legislations or might result in divergent conclusions.

The outcome of this screening approach reveals that substances for which safe use in humans is known from decades of experience might possibly be classified as endocrine dis- ruptors with yet unknown regulatory consequences. Such a classification might frighten and unsettle consumers. This emphasizes the need for elaborating more examples (including more naturally occurring substances) in order to be able to understand the practical consequences of any set of criteria for regulating substances with ED properties.

\section{Conclusion}

The WHO/IPCS definition for endocrine disruptors is a general accepted and suitable basis for the identification of endocrine disruptors. Further the OECD Guidance Document on Standardized Test Guidelines for Evaluating Chemicals for Endocrine Disruption [2] is very helpful in identifying EATS mediated effects. But further guidance on the identification and interpretation of other non-EATS mediated effects is necessary.

The WHO definition of adverse effects provides a framework for the interpretation of effects, but the examples in this screening activity showed that in-depth assessments are required to respond to the criteria for EATS-mediated effects. Also, substance specific evaluations and interpretations will challenge the evaluators in the future.

To create a plausible link between the endocrine mediated adverse effect and the endocrine mode of action may be challenging, especially when different MoA might be possible for a certain effect and if the investigations on the adverse effect and the MoA are performed in different studies. Future evaluations will show if such a relation can unequivocally be established for the substances of interest or if there remains uncertainty. Substances, for which such a relation could not be clearly demonstrated will not be classified according to the current EC proposal for identification of ED under biocide and pesticide regulation. This might be unsatisfying, as substances for which no such link can be unequivocally demonstrated remain unregulated. It has to be questioned if an approach with different levels of evidence, as included in EC option 3, presented in the roadmap would be more appropriate.

This screening exercise shows that substances occurring naturally and which are available at relevant amounts in our food, such as genistein and caffeine, would be classified as ED by all (genistein) or most (caffeine) of the sets of criteria applied. Potency considerations as proposed by BfR [47] would avoid classification of caffeine. These examples increase our understanding of the consequences of applying the various sets of criteria under discussion.

\section{List of abbreviations}

AGD: Anogenital distance

Bw: Body weight

Cefic: European Chemical Industry Council

EATS: Estrogen, androgen, thyroid, steroidogenesis

EC: European Commission

ECPA: European Crop Protection Association

ED: Endocrine disruptor

EFSA: European Food Safety Authority

ER: Estrogen receptor

GD: Guidance Document 
F: Female

JRC: Joint Research Centre of the European Commission

IARC: International Agency for Research on Cancer

LOAEL: Lowest observed adverse effect level

M: Male

MoA: Mode of action

NOAEL: No observed adverse effect level

NTP: National Toxicology Program

OECD: Organisation for Economic Co-operation and Development

PND: Postnatal day

Ppm: Parts per million

RACB: Reproductive assessment by continuous breeding

REACH: Registration, Evaluation, Authorisation, and Restriction of Chemicals

STOT: Specific target organ toxicity

TG: Test Guideline

Vit-D3: Vitamin D3

\section{Competing interests}

The authors declare that they have no competing interests.

\section{Authors' contributions}

\begin{tabular}{|l|c|c|c|}
\hline Authors' contributions & USW & JUV & KS \\
\hline Research concept and design & $\checkmark$ & -- & $\checkmark$ \\
\hline Collection and/or assembly of data & $\checkmark$ & $\checkmark$ & -- \\
\hline Data analysis and interpretation & $\checkmark$ & $\checkmark$ & -- \\
\hline Writing the article & $\checkmark$ & -- & -- \\
\hline Critical revision of the article & $\checkmark$ & $\checkmark$ & $\checkmark$ \\
\hline Final approval of article & $\checkmark$ & -- & -- \\
\hline Statistical analysis & -- & -- & -- \\
\hline
\end{tabular}

\section{Acknowledgements}

This project was sponsored by Cefic (European Chemical Industry Council) and ECPA (European Crop Protection Association). Their support is greatly acknowledged. However, this publication only represents the personal opinion of the authors and not the opinion of Cefic or ECPA.

Publication history

Editor: John E. Doe, Parker Doe Partnership LLP, UK.

Received: 15-Dec-2016 Final Revised: 23-Feb-2017

Accepted: 21-Apr-2017 Published: 02-May-2017

\section{References}

1. EC, European Commission. COMMISSION STAFF WORKING DOCUMENT. IMPACT ASSESSMENT. Defining criteria for identifying endocrine disruptors in the context of the implementation of the plant protection products regulation and biocidal products regulation. Main report. Accompanying the document COMMUNICATION FROM THE COMMISSION TO THE EUROPEAN PARLIAMENT AND THE COUNCIL on endocrine disruptors and the draft Commission acts setting out scientific criteria for their determination in the context of the EU legislation on plant protection products and biocidal products. Brussels, Belgium. 2016. | Pdf

2. OECD, Organisation for Economic Co-Operation and Development. GUIDANCE DOCUMENT ON STANDARDISED TEST GUIDELINES FOR EVALUATING CHEMICALS FOR ENDOCRINE DISRUPTION. Series on Testing and Assessment. No. 150. ENV/JM/MONO(2012)22. 2012. | Pdf

3. EC, European Commission: ROADMAP: Defining criteria for identifying Endocrine Disruptors in the context of the implementation of the Plant Protection Product Regulation and Biocidal Products Regulation. DG ENV.A.3, DG SANCO.E.3. 2014.

4. CEFIC, Conseil Européen des Fédérations de l'Industrie Chimique.
Proposal for criteria for identification of endocrine disruptors. 2014. | Website

5. WHO/IPCS, World Health Organization/International Programme on Chemical Safety: Global Assessment of the State-of-the-Science of Endocrine Disruptors. WHO/PCS/EDC/02.2. WHO, World Health Organization, Geneva. 2002. I Pdf

6. EC, European Commission. Impact assessment on criteria to identify endocrine disruptors - technical meeting on the Joint Research Center methodology. European Commission, DG Health and Food Safety, Public health, Endocrine Disruptors. 2015. | Article

7. ACGIH, American Conference of Governmental Industrial Hygienists. Sucrose. In (Eds.), Documentation of the Threshold Limit Values and Biological Exposure Indices, 7th Ed., 2011 2001, 3.

8. Tinwell $\mathrm{H}$, Colombel $\mathrm{S}$, Blanck $\mathrm{O}$ and Bars $\mathrm{R}$. The screening of everyday life chemicals in validated assays targeting the pituitary-gonadal axis. Regul Toxicol Pharmacol. 2013; 66:184-96. | Article | PubMed

9. Rozman KK, Bhatia J, Calafat AM, Chambers C, Culty M, Etzel RA, Flaws JA, Hansen DK, Hoyer PB, Jeffery EH, Kesner JS, Marty S, Thomas JA and Umbach D. NTP-CERHR expert panel report on the reproductive and developmental toxicity of genistein. Birth Defects Res B Dev Reprod Toxicol. 2006; 77:485-638. | Article | PubMed Abstract | PubMed FullText

10. NTP, National Toxicology Program. Multigenerational Reproductive Toxicology Study of Genistein (CAS: No. 446-72-0) in Sprague-Dawley Rats (Feed Study). NTP TR 539. U.S. Department of Health and Human Services, Public Health Services, National Institutes of Health. | Pdf

11. Latendresse JR, Bucci TJ, Olson G, Mellick P, Weis CC, Thorn B, Newbold $\mathrm{RR}$ and Delclos KB. Genistein and ethinyl estradiol dietary exposure in multigenerational and chronic studies induce similar proliferative lesions in mammary gland of male Sprague-Dawley rats. Reprod Toxicol. 2009; 28:342-53. | Article | PubMed

12. McClain RM, Wolz E, Davidovich A, Pfannkuch F and Bausch J. Subchronic and chronic safety studies with genistein in dogs. Food Chem Toxicol. 2005; 43:1461-82. | Article | PubMed

13. Michael McClain R, Wolz E, Davidovich A, Pfannkuch F, Edwards JA and Bausch J. Acute, subchronic and chronic safety studies with genistein in rats. Food Chem Toxicol. 2006; 44:56-80. | Article | PubMed

14. NTP, National Toxicology Programme. Toxicology and Carcinogenesis Studies of Genistein (CAS No. 446-72-0) in Sprague-Dawley Rats (Feed Study). NTP TR 545. U.S. Department of Health and Human Services, Public Health Services, National Institutes of Health. 2007. I Pdf

15. Li Y, Luh CJ, Burns KA, Arao Y, Jiang Z, Teng CT, Tice RR and Korach KS. Endocrine-Disrupting Chemicals (EDCs): In Vitro Mechanism of Estrogenic Activation and Differential Effects on ER Target Genes. Environ Health Perspect. 2013; 121:459-66. | Article I PubMed Abstract I PubMed FullText

16. BfR, Bundesinstitut für Risikobewertung. Joint DE - UK Position Paper Regularory definition of an endocrine disrupter in relation to potential threat to human health. Proposal applicable in the regulatory context of Plant Protection Products, Biocidal Products, and Chemicals targeted by REACH. 2011. I Pdf

17. Lee BJ, Jung EY, Yun YW, Kang JK, Baek IJ, Yon JM, Lee YB, Sohn HS, Lee JY, Kim KS and Nam SY. Effects of exposure to genistein during pubertal development on the reproductive system of male mice. J Reprod Dev. 2004; 50:399-409. | PubMed

18. Moltmann JF, Liebig M, Knacker T, Keller M, Scheurer M and Ternes T. Gewässerrelevanz endokriner Stoffe und Arzneimittel. Neubewertung des Vorkommens, Erarbeitung eines Monitoringkonzepts sowie Ausarbeitung von Maßnahmen zur Reduzierung des Eintrags in Gewässer [Relevance of endocrine disrupting substances and pharmaceuticals in surface waters]. Im Auftrag des Umweltbundesamtes im Rahmen des Umweltforschungsplanes-FKZ 20524 205. 2007. | Pdf

19. Sassi-Messai S, Gibert Y, Bernard L, Nishio S, Ferri Lagneau KF, Molina J, Andersson-LendahI M, Benoit G, Balaguer P and Laudet V. The phytoestrogen genistein affects zebrafish development through two different pathways. PLoS One. 2009; 4:e4935. | Article | PubMed Abstract | PubMed FullText

20. Stevenson LM, Brown AC, Montgomery TM and Clotfelter ED. 
Reproductive consequences of exposure to waterborne phytoestrogens in male fighting fish Betta splendens. Arch Environ Contam Toxicol. 2011; 60:501-10. | Article | PubMed

21. Rearick DC, Fleischhacker NT, Kelly MM, Arnold WA, Novak PJ and Schoenfuss HL. Phytoestrogens in the environment, I: occurrence and exposure effects on fathead minnows. Environ Toxicol Chem. 2014; 33:553-9. | Article | PubMed

22. Brown AC, Stevenson LM, Leonard HM, Nieves-Puigdoller K and Clotfelter ED. Phytoestrogens beta -sitosterol and genistein have limited effects on reproductive endpoints in a female fish, Betta splendens. Biomed Res Int. 2014; 2014:681396. | Article | PubMed Abstract | PubMed FullText

23. EFSA. Scientific Opinion on the safety of caffeine. The EFSA Journal 2015; 13:1-120.

24. IARC, International Agency for Research on Cancer: IARC Monographs on the Evaluation of Carcinogenic Risks to Humans. Coffee, Tea, Mate, Methylxanthines and Methylglyoxal. WHO, World Health Organization, Geneva. 1991. 51. I Website

25. OECD, Organisation for Economic Co-Operation and Development. SIDS Initial Assessment Profile for SIAM 14 (Paris, France, 26-28 March 2002). Caffeine. 2002. I Website

26. NTP, National Toxicology Program. Final Report on the Reproductive Toxcity of Caffeine (CAS No. 58-08-2) Administered by Gavage to Sprague-Dawley Rats. RACB94006. PB86-211743. U.S. Department of Health and Human Services, Public Health Services, National Institutes of Health. 1996.

27. Sarobo C, Lacorte LM, Martins M, Rinaldi JC, Moroz A, Scarano WR, Delella FK and Felisbino SL. Chronic caffeine intake increases androgenic stimuli, epithelial cell proliferation and hyperplasia in rat ventral prostate. Int J Exp Pathol. 2012; 93:429-37. | Article | PubMed Abstract | PubMed FullText

28. Oluwole OF, Salami SA, Ogunwole E and Raji Y. Implication of caffeine consumption and recovery on the reproductive functions of adult male Wistar rats. J Basic Clin Physiol Pharmacol. 2016; 27:483-91. | Article | PubMed

29. EPA, Environmental Protection Agency: Guidelines for Reproductive Toxicity Risk Assessment. U.S. Environmental Protection Agency, Washington, DC. 1996. | Website

30. Ross AC, Taylor CL, Yaktine AL and Del Valle HB. Dietary Reference Intakes for Calcium and Vitamin D. IOM,, Institute of Occupational Medicine. Committee to Review Dietary Reference Intakes for Vitamin $\mathrm{D}$ and Calcium. Food and Nutrition Board. National Academies Press, Washington, DC. 2011. I Website

31. Tischler AS, Powers JF, Pignatello M, Tsokas P, Downing JC and McClain RM. Vitamin D3-induced proliferative lesions in the rat adrenal medulla. Toxicol Sci. 1999; 51:9-18. | PubMed

32. Shephard RM and Deluca HF. Plasma concentrations of vitamin D3 and its metabolites in the rat as influenced by vitamin D3 or 25-hydroxyvitamin D3 intakes. Arch Biochem Biophys. 1980; 202:43-53. I PubMed

33. ECHA, European Chemicals Agency. CLH report. Proposal for Harmonised Classification and Labelling. Based on Regulation (EC) No 1272/2008 (CLP Regulation), Annex VI, Part 2. International Chemical Identification: Colecalciferol, Vitamin D3. | Website

34. EFSA, European Food Safety Authority. Scientific Opinion on the Tolerable Upper Intake Level of vitamin D. The EFSA Journal. 2012; 10:1-45

35. McClain RM, Langhoff L and Hoar RM. Reproduction studies with 1 alpha, 25-dihydroxyvitamin D3 (calcitriol) in rats and rabbits. Toxicol Appl Pharmacol. 1980; 52:89-98. | PubMed

36. Deluca HF, Prahl JM and Plum LA. 1,25-Dihydroxyvitamin $D$ is not responsible for toxicity caused by vitamin $D$ or 25 -hydroxyvitamin $D$. Arch Biochem Biophys. 2011; 505:226-30. | Article | PubMed

37. Swami S, Krishnan AV, Peng L, Lundqvist J and Feldman D. Transrepression of the estrogen receptor promoter by calcitriol in human breast cancer cells via two negative vitamin $D$ response elements. Endocr Relat Cancer. 2013; 20:565-77. | Article | PubMed

38. EFSA, European Food Safety Authority and ANS, Panel on Food Additives and Nutrient Sources Added to Food. Risk assessment for peri- and post-menopausal women taking food supplements containing isolated isoflavones. The EFSA Journal. 2015; 13:342. | Pdf

39. Krishnan AV, Swami S and Feldman D. Vitamin D and breast cancer: inhibition of estrogen synthesis and signaling. J Steroid Biochem Mol Biol. 2010; 121:343-8. | Article | PubMed

40. Krishnan AV, Swami S, Peng L, Wang J, Moreno J and Feldman D. Tissueselective regulation of aromatase expression by calcitriol: implications for breast cancer therapy. Endocrinology. 2010; 151:32-42. | Article | PubMed Abstract | PubMed FullText

41. Swami S, Krishnan AV, Wang JY, Jensen K, Peng L, Albertelli MA and Feldman D. Inhibitory effects of calcitriol on the growth of MCF-7 breast cancer xenografts in nude mice: selective modulation of aromatase expression in vivo. Horm Cancer. 2011; 2:190-202. | Article | PubMed Abstract | PubMed FullText

42. Daly M. Sugars, insulin sensitivity, and the postprandial state. Am J Clin Nutr. 2003; 78:865S-872S. | Article | PubMed

43. Gibson S, Gunn P, Wittekind A and Cottrell R. The effects of sucrose on metabolic health: a systematic review of human intervention studies in healthy adults. Crit Rev Food Sci Nutr. 2013; 53:591-614. | Article | PubMed Abstract | PubMed FullText

44. FDA, Food and Drug Administration. Select Committee on GRAS Substances (SCOGS) Opinion: Sucrose. 2015. I Website

45. SCPAFF, Standing Committee on Plants, Animals, Food and Feed. Review report for the basic substance sucrose. Final. SANCO/11406/2014rev. 2, 11 July 2014. European Commission, Health and Consumers Directorate General, Safety of the Food Chain, Chemicals, contaminants, pesticides. Brussels, Belgium. Fisportal. 2014.

46. Solecki R, Kortenkamp A, Bergman A, Chahoud I, Degen GH, Dietrich $D$, Greim H, Hakansson H, Hass U, Husoy T, Jacobs M, Jobling S, Mantovani A, Marx-Stoelting P, Piersma A, Ritz V, Slama R, Stahlmann $R$, van den Berg M, Zoeller RT and Boobis AR. Scientific principles for the identification of endocrine-disrupting chemicals: a consensus statement. Arch Toxicol. 2017; 91:1001-1006. | Article | PubMed Abstract | PubMed FullText

47. BfR, Bundesinstitut für Risikobewertung. Scientific principles for the identification of endocrine disrupting chemicals - a consensus statement. Outcome of an international expert meeting organized by the German Federal Institute for Risk Assessment (BfR). 2016.

48. Slama R, Bourguignon JP, Demeneix B, Ivell R, Panzica G, Kortenkamp A and Zoeller RT. Scientific Issues Relevant to Setting Regulatory Criteria to Identify Endocrine-Disrupting Substances in the European Union. Environ Health Perspect. 2016; 124:1497-1503. | Article | PubMed Abstract | PubMed FullText

\section{Citation:}

Schuhmacher-Wolz U, Voss J-U and Schneider K. Case study with natural substances on the various options to identify and categorise endocrine disruptors. J Toxicol Health. 2017; 4:2.

http://dx.doi.org/10.7243/2056-3779-4-2 